\title{
Age-Related Differences in Temporal and Spatial Dimensions of Episodic Memory Performance Before and After Hundred Days of Practice
}

\author{
Hannes Noack \\ Max Planck Institute for Human Development, Berlin, Germany, \\ and Tübingen University
}

Florian Schmiedek

Max Planck Institute for Human Development, Berlin, Germany, and German Institute for International Educational Research, Frankfurt am Main, Germany

\author{
Martin Lövdén \\ Max Planck Institute for Human Development, Berlin, Germany, \\ and Karolinska Institutet \& Stockholm University \\ Ulman Lindenberger \\ Max Planck Institute for Human Development, Berlin, Germany
}

\begin{abstract}
Normal aging impairs the representation and integration (binding) of spatial and temporal context in episodic memory. We directly compare age differences in episodic memory in relation to processing spatial and temporal context. As part of the COGITO study, 101 younger and 103 older participants trained an object-location serial recall task for 100 sessions. Training exacerbated the recall deficit of older relative to younger adults. Younger adults improved in recall performance on both spatial and temporal dimensions. In contrast, older adults improved on the spatial dimension only. Individual differences in pretest performance and change were positively correlated across dimensions among younger adults but negatively related among older adults. We conclude that older adults are impaired at simultaneously processing spatial and temporal context and preferentially process spatial at the expense of temporal context.
\end{abstract}

Keywords: aging, training, context binding, latent change score model, representational precision

Episodic memories include information about the content of an event, such as the name of a person, and context, such as where and when this person has been met (Johnson, Hashtroudi, \& Lindsay, 1993; Light, 1991; Tulving, 1983). Cross-sectional (Cansino, 2009; Nilsson et al., 1997; Salthouse, 1998) and longitudinal (de Frias, Lövdén, Lindenberger, \& Nilsson, 2007; Rönnlund, Nyberg, Bäckman, \& Nilsson, 2005) research has shown that episodic memory performance declines with adult age. In general, older individuals have particular difficulties in recollecting contextual information, whereas memory for content is less affected

This article was published Online First February 25, 2013.

Hannes Noack, Center for Lifespan Psychology, Max Planck Institute for Human Development, Berlin, Germany, and Institute for Medical Psychology and Behavioral Neurobiology, Tübingen University, Tübingen, Germany; Martin Lövdén, Center for Lifespan Psychology, Max Planck Institute for Human Development, Berlin, Germany, and Aging Research Center, Karolinska Institutet \& Stockholm University, Stockholm, Sweden; Florian Schmiedek, Center for Lifespan Psychology, Max Planck Institute for Human Development, Berlin, Germany, and Center for Research on Education and Human Development (DIPF), German Institute for International Educational Research, Frankfurt am Main, Germany; Ulman Lindenberger, Center for Lifespan Psychology, Max Planck Institute for Human Development, Berlin, Germany.

Correspondence concerning this article should be addressed to Hannes Noack, Center for Lifespan Psychology, Max Planck Institute for Human Development, Lentzeallee 94, 14195 Berlin, Germany. E-mail: noack@ mpib-berlin.mpg.de
(Spencer \& Raz, 1994, 1995). However, several findings challenge such a general conclusion and ask for detailed analysis of the characteristics of context, including how it relates to content (Spencer \& Raz, 1995). For example, Chalfonte and Johnson (1996) segmented complex memories into memory for features (e.g., item identity, spatial, and color context) and memory for associations (i.e., binding) between features. When assessing these single features in separation, the authors found age-related differences in memory for location but not in memory for identity or color. Thus, age-related differences in source memory depend on the characteristics of the feature dimension. In addition, Chalfonte and Johnson (1996) also observed that feature binding is particularly impaired in older adults (see also Bayen, Phelps, \& Spaniol, 2000; Mitchell, Johnson, Raye, Mather, \& D'Esposito, 2000; Naveh-Benjamin, 2000; Naveh-Benjamin et al., 2009; Shing et al., 2010; Shing, Werkle-Bergner, Li, \& Lindenberger, 2008).

Spatial and temporal coordinates are the most prominent aspects of context (e.g., Brown \& McCormack, 2006; Gallistel, 1990; Kahneman, Treisman, \& Gibbs, 1992; Tulving, 1983; Xu \& Chun, 2009). In a seminal article, Hasher and Zacks (1979) argued that age-related differences in memory for both spatial and temporalorder information were minor because such information is processed automatically. In fact, several attempts to demonstrate age-related decrements in spatial location memory have failed (Meulenbroek et al., 2010; Mitchell et al., 2000; Olson et al., 2004; Parkin, Walter, \& Hunkin, 1995; Vakil \& Tweedy, 1994). At the same time, other studies have observed age-related deficits in spatial location processing (Chalfonte \& Johnson, 1996; Cherry \& 
Park, 1993; Kessels, Hobbel, \& Postma, 2007; Naveh-Benjamin, 1987, 1988; Perlmutter, Metzger, Nezworski, \& Miller, 1981; Puglisi, Park, Smith, \& Hill, 1985). These age-related differences appear to be further amplified when binding demands are present (e.g., Chalfonte \& Johnson, 1996; Mitchell et al., 2000; Kessels et al., 2007; Old \& Naveh-Benjamin, 2008).

Research on age differences in temporal-order memory has been less equivocal than research on age differences in location memory. With the exception of a few studies (Perlmutter et al., 1981; Vakil, Weise, \& Enbar, 1997), temporal-order memory has been found to deteriorate with advancing age. Age-related differences have been observed in recency judgment tasks (Fabiani \& Friedman, 1997; Parkin et al., 1995), quarter of the list judgments tasks (Vakil \& Tweedy, 1994), list learning tasks (Naveh-Benjamin, 1990; Vakil et al., 1997), as well as serial (Golomb, Peelle, Addis, Kahana, \& Wingfield, 2008; Kessels et al., 2007; Li et al., 2010) and free (Golomb et al., 2008; Kahana, Howard, Zaromb, \& Wingfield, 2002) recall tasks. Note, however, that these tasks, in contrast to the typical measures of location memory, require the binding of information between an item and its serial position. These binding demands may aggravate age differences in performance.

Going beyond measures of mean accuracy, serial position curves have been a useful tool for analyzing temporal-order memory (Lewandowsky \& Farrell, 2008; Richardson, 2007). These curves typically show higher recall performance at early and late list positions, with lower recall performance at intermediate list positions. Notably, age-related differences in recall performance are most pronounced at these intermediate parts of the serial position curve (Golomb et al., 2008; Li et al., 2010; Maylor, Vousden, \& Brown, 1999; Naveh-Benjamin, Cowan, Kilb, \& Chen, 2007), which has been attributed to an age-related decline in the ability to represent temporal order. According to theoretical concepts of the representation of temporal order, which assume a central role for the association between item information and a continually changing temporal context (Howard, Kahana, \& Wingfield, 2006; Kahana et al., 2002; but see Oberauer \& Lewandowsky, 2008, for a discussion of alternative accounts), these age-related differences may be another expression of the associative deficit in the old (Golomb et al., 2008). Because serial position curves are more sensitive to the representations of temporal order than to representations of other stimulus dimensions (e.g., spatial location; van Asselen, Van der Lubbe, \& Postma, 2006), we may predict a stronger effect of serial position on the retrieval of temporal order information than on retrieval of other stimulus dimensions, which then should be stronger in the older than in the younger group. Note that the temporal organization of list items we are referring to here is defined in terms of the interrelation of discrete events rather than pointers on a continuous representation of time (cf. Lewandowsky, Brown, Wright, \& Nimmo, 2006; Oberauer \& Lewandowsky, 2008).

Few studies have directly compared age differences in temporal and spatial memory, and even fewer studies have analyzed the interactions between the processing of these types of context. Kessels and colleagues (2007) contrasted temporal binding and spatial binding using serial reconstruction and spatial recall paradigms. Age-related differences in performance were of similar magnitude for both sources, indicating a general decrease in temporal and spatial source memory with advancing age. However, behavioral (Hälbig, Mecklinger, Schriefers, \& Friederici, 1998; Dutta \& Nairne, 1993; Parkin et al., 1995; van Asselen et al., 2006), neuroimaging (Duarte, Henson, Knight, Emery, \& Graham, 2010; Ekstrom, Copara, Isham, Wang, \& Yonelinas, 2011), electroencephalographic (EEG; Hsieh, Ekstrom, \& Ranganath, 2011), and lesion (Kopelman, Stanhope, \& Kingsley, 1997) studies suggest that spatial and temporal order context is processed independently to some degree. Specifically, temporal retrieval tasks may be more dependent of the prefrontal cortex than spatial retrieval tasks. Performance deficits in older relative to younger adults tend to be particularly pronounced in tasks that critically depend on the frontal lobes (Braver et al., 2001; Craik, Luo, \& Sakuta, 2010; Lindenberger, Burzynska, \& Nagel, in press; West, 1996), further motivating inquiries into the relative magnitude of age differences in spatial and temporal source memory. In addition, the interaction between the two types of processing is poorly understood. Given that temporal and spatial coordinates define events that, in turn, form the basis of episodic memories, content may be encoded in relation to both, either one, or none of these two context dimensions, and the relative proportion of these alternative options may vary between individuals and situations, and may change with age. For example, it has been shown that individuals can focus on the temporal while ignoring the spatial dimension and vice versa (Dutta \& Nairne, 1993; van Asselen et al., 2006), and that focusing on both dimensions simultaneously can have detrimental effects on performance (Dutta \& Nairne, 1993).

Cognitive aging researchers also take interest in improving episodic memory performance through training (for reviews, see Hertzog, Kramer, Wilson, \& Lindenberger, 2009; Lövdén, Bäckman, Lindenberger, Schaefer, \& Schmiedek, 2010; Noack, Lövdén, Schmiedek, \& Lindenberger, 2009). Kliegl, Smith, and Baltes (1989; Baltes \& Kliegl, 1992) tested younger and older participants at pretest, after mnemonic strategy instruction, and after additional extensive practice. Although both age groups gained substantially from training, age-related differences at pretest were magnified after training, indicating age-related differences in training gains. Several more recent age-comparative intervention studies using strategy instruction (Brehmer, Li, Müller, von Oertzen, \& Lindenberger, 2007; Shing et al., 2008) or process-oriented extensive training (Schmiedek, Lövdén, \& Lindenberger, 2010) have confirmed these findings. However, the extent to which agerelated differences in training gains are different for memory of temporal and spatial context remains unknown. Moreover, individual and age group differences in the associations between improvements in the two dimensions, which are both key components of measures of complex episodic memory performance, have not been studied thus far. Such differences may be expected based on findings that focusing on both dimensions simultaneously can have detrimental effects on performance (Dutta \& Nairne, 1993).

Finally, we note that spatial and temporal context memory performance is typically evaluated with strict coding rules (i.e., responses are considered correct if the original value is reproduced and wrong if otherwise). This procedure implicitly builds on the assumption of all-or-none representations, where items are either encoded correctly with the correct item coordinates or not at all. That is, errors are regarded as random. However, it seems more realistic to conceive of representations of time and space as probability distributions. For example, spatial positions may have a certain probability value assigned to it, such that several neigh- 
boring sites are plausible for a particular object (Noack, Lövdén, \& Lindenberger, 2012). This assumption has its antecedents in the concept of discriminal distributions (cf. Thurstone, 1927), in signal detection theory (e.g., Wickens, 2002; see also Sanborn, Griffiths, \& Shiffrin, 2010), and-on a more abstract level-in the discussion of partial source memory (Dodson, Holland, \& Shimamura, 1998; Johnson et al., 1993; Simons, Dodson, Bell, \& Schacter, 2004; Slotnick \& Dodson, 2005). Adopting this view, errors may be informative about the precision of the underlying representations and provide more information about age differences in episodic memory. Note, however, that the idea of partial source memory does not exclude the possibility that items and its coordinates may be forgotten.

In summary, episodic memories are defined in terms of various context dimensions, such as temporal and spatial coordinates of any specific event. Completely correct encoding and retrieval requires binding between these strands of information. Relative age differences in memory performance for spatial and temporal context dimensions have rarely been studied and directly compared, and individual differences in the interactions between these two dimensions of episodic memory have largely been ignored. This study seeks to fill these gaps by investigating age-related differences in episodic memory performance in a task that requires integration of object, temporal order, and spatial location information (see Figure 1). We determine overall accuracy, the accuracy of binding locations to objects, and the accuracy of binding temporal order to objects, and study the magnitude of age-related differences in these components of episodic memory. Data originated from COGITO (Schmiedek, Lövdén, et al., 2010), a large-scale intervention study comprising the assessment of 103 older and 101 younger participants over more than 100 sessions of training. The relatively large sample of this study allowed us to investigate whether performance on the spatial and temporal aspects of the

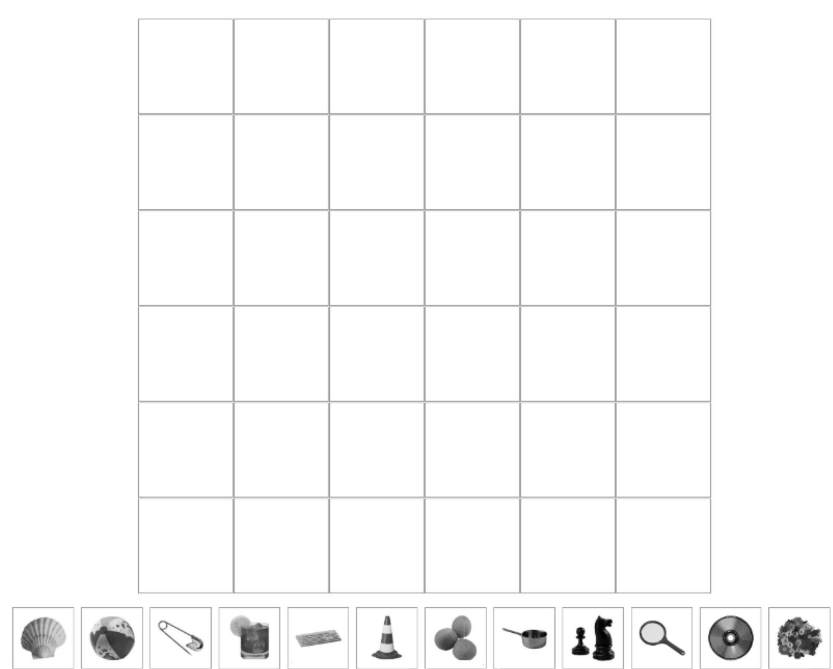

Figure 1. Example of stimulus display at test. Participants selected objects with mouse clicks and placed it at the corresponding position using the mouse pointer and clicking on to the respective cell. Selected objects disappeared from the list at the bottom. At study, objects were presented one-by-one at different locations. Only one object was visible at a time and no list of objects was presented at the bottom. task is differentially correlated in younger and older adults. Furthermore, the extensive practice period of the study made it possible to study age differences in practice-related improvements in temporal and spatial accuracy, as well as the associations between individual differences in practice-related improvements on these two dimensions. Based on the literature reviewed here, we expected to see age-related differences on all dependent measures at pretest and at posttest. As the literature is ambiguous with respect to the effects of aging on temporal and spatial information processing, however, we studied this issue exploratorily. Finally, we studied erroneous responses and contrasted them with the expected pattern of errors based on a random guessing model to investigate whether memory representations are better thought of in terms of probability distributions. The latter analyses also provided information about the precision of the underlying representations as a function of age and practice. As noted, we expected to see less precise representations in the elderly.

\section{Methods}

The COGITO study (Schmiedek, Bauer, Lövdén, Brose, \& Lindenberger, 2010; Schmiedek, Lövdén, et al., 2010) was conducted at the Max Planck Institute for Human Development in Berlin, Germany. In the main study, 103 older and 101 younger participants were tested on cognitive tasks during more than 100 daily sessions. The daily cognitive battery consisted of 12 different tasks tapping into different cognitive abilities (working memory, episodic memory, and perceptual speed) in different content domains (verbal, spatial-figural, and numerical). The present work focuses on age-related differences at baseline and in practicerelated changes in object-position memory, a task that we classified as assessing episodic memory in the spatial-figural domain. For a description of the other tasks, see Schmiedek, Bauer, et al. (2010).

\section{Participants}

One hundred one younger ( $51.5 \%$ women; age $=20$ to 31 years $)$ and 103 older ( $49.5 \%$ women, age $=65$ to 80 years) adults participated in the entire study (see Table 1 for sample description). Seven younger adults and 10 older adults withdrew from the study during pretest. Attrition during the training period was low (19 younger adults and 5 older adults). Participants were paid between 1,450 and 1,950 euros for their participation, depending on the number and pace of completed sessions during the training period of the study. As described in more detail in Schmiedek, Lövdén, et al. (2010), the sample was similar to samples of healthy younger and older adults in research on cognitive aging.

\section{Object-Position Memory Task}

Twelve colored photographs of real-world objects were presented sequentially at different locations in a $6 \times 6$ cell grid (see Figure 1). Items were presented in immediate succession for 1,000 , $2,000,4,000$, or $8,000 \mathrm{~ms}$. The retrieval phase began $1 \mathrm{~s}$ after the presentation sequence had ended. All 12 presented objects appeared simultaneously in random order below the empty grid. Participants were instructed to select the objects in the right order and place them at the corresponding positions using the computer 
Table 1

Sample Means (SDs)

\begin{tabular}{lcc}
\hline & Younger & Older \\
\hline Age (years) & $25.6(2.7)$ & $71.3(4.1)$ \\
Years of education & $16.1(3.2)$ & $13.6(3.6)$ \\
WAIS Digit-Symbol & $60.3(9.5)$ & $43.6(9.0)$ \\
Spot-a-Word Test & $0.66(0.1)$ & $0.81(0.1)$ \\
\hline
\end{tabular}

Note. $\quad$ WAIS $=$ Wechsler Adult Intelligence Scale.

mouse. Each response was confirmed by selection of another object from the list. After having selected a new object, the former item disappeared from the grid and from the list, and corrections were no longer possible.

\section{Procedure}

The study was designed as a pretest-posttest intervention study. At pretest and posttest, all participants completed a total of 10 sessions of 2 to $2.5 \mathrm{hr}$ each, comprised of a comprehensive battery of computerized and paper-pencil cognitive tests. Twenty-four trials of the object-position memory were included in the pretestposttest battery. During the training period, participants completed 101 training sessions (younger adults: $M=100.8, S D=2.6$, range $=87$ to 109 ; older adults: $M=101.0, S D=2.7$, range $=$ 90 to 106) over an average period of 197 and 188 days for younger and older adults, respectively. Testing and training took place in lab rooms in groups of no more than 6 participants.

All participants practiced the object position task in 12 warm-up trials, with three trials at each of the four presentation times, which were always given in decreasing order. During pretest and posttest, 24 trials of the object-position memory task were administered in blocks of three trials with the same presentation time. The order of presentation times-8,000, 4,000, 2,000, 1,000, 1,000, 2,000, 4,000, 8,000 ms-was constant across measurement time points and individuals. Two trials of the object-position memory task were presented at each training session. Different from pre- and posttest, presentation times were assigned individually based on pretest performance. Individual demand levels were kept constant over the entire training period. Calibration of presentation times served to create optimal levels of demand in all participants and to avoid excessive demands as well as boredom (see Schmiedek, Bauer, et al., 2010, for details).

\section{Data Analysis}

Scoring of correct responses. Three measures of accuracy were established: overall, temporal, and spatial accuracy. For overall accuracy, a given response was assigned a value of 1 on overall accuracy when the correct object was chosen at the correct serial position and placed at the correct spatial location in the grid, and assigned a value of 0 otherwise. Similarly, we scored spatial and temporal accuracy as 1 when the respective characteristic of the item was correctly reproduced, irrespective of the other dimension. Accordingly, spatial and temporal accuracies were scored 0 when an object was placed in the wrong cell or chosen at the wrong time, respectively. ${ }^{1}$ To analyze how well accuracy measures in both context dimensions overlapped to form overall ac- curacy, we determined the proportion of overall accurate trials over trials where at least one dimension was correctly recalled.

Scoring and analysis of spatial errors. First, spatial error magnitude was determined for each erroneous response by taking the Euclidean distance between response location and original location of a selected object. Next, we constructed a reference data set of expected random distances for each erroneous response. This was done because the maximal and expected random distances depend on the original object location and the distribution of original object locations may differ between individuals as well as between time points. Observed and simulated error distances can then be compared by graphical means and the differences between both measures can be used as corrected measures of error distance.

Expected random displacements equaled the mean distance between the original location and every other spatial location in the grid. Inspection of the spatial distributions of erroneous responses, however, revealed that participants tended to not select the corners of the $6 \times 6$ grid. Thus, determining expected random displacement based on all possible locations might have produced values that were too high. We, therefore, constructed another data set based on the strong assumption that participants strategically chose one of the nine innermost grid cells whenever spatial information was not available. Note that this strategy strongly reduced expected random displacement and may therefore be considered a conservative test, giving information on the lower bound of the expected random displacement.

Scoring and analysis of temporal errors. First, we determined the magnitude of temporal error for each erroneous response by taking the absolute distance between the original and the response serial position of a selected item. The expected temporal distance depends on the original serial position as well as the number and serial position of other correctly recalled items. For example, the temporal distance of an item that had been presented at serial position five, may take values of no more than 7 , whereas an item that had been presented at serial position 12 may take values of no more than 11 . If all but the 11th and 12th item were recalled correctly in the latter case, however, temporal distance may take values of no more than one. We constructed a data set of expected temporal distances because the distribution of these aspects likely differed between individuals and time points.

Expected temporal distance was determined for each response separately, taking the serial position and the set of available alternative serial positions within the corresponding trial into account.

Analysis of group differences and changes. We analyzed the effects of age and session on overall, temporal, and spatial accuracy using linear mixed effects models (lmer of package lme4, Bates \& Maechler, 2010, in R, R Development Core Team, 2010) with heterogeneous variance-covariance structures, both within

\footnotetext{
${ }^{1}$ It has been suggested in the literature (Maylor, Vousden, \& Brown, 1999) that older participants may be disadvantaged by strict scoring rules in temporal order memory, because they are more likely to commit omissions early in the sequence. To address this point, we determined Spearman's rank correlation coefficient between the original and the response item sequence. This measure assesses relative ordering in the sequence and is therefore less sensitive to early errors. Correlations between strict temporal accuracy and Fisher's Z transformed Spearman's rho were high, $r>$ .89 , and analyses of mean differences were similar for both measures.
} 
and between age groups. To investigate mean-level differences between the two context dimensions and their differences over age groups and measurement occasions, we further tested a model comprising context dimension as a factor and spatial and temporal accuracy data as dependent measure. We corrected spatial and temporal accuracy for guessing rates $($ spatial $=1 / 36$; temporal $=$ 1/12) to warrant comparability. Significance of effects was addressed using model comparisons according to Type III sums of squares, where reduced models (with single term deletion) are contrasted against the full model. Consequently, main effects reflect the average effect of a factor in the presence of the main effects of and interactions with all other factors. Mean differences in serial positions curves were analyzed on the basis of aggregated data. Curves were split into three parts: primacy-containing serial positions 1 to 3 ; intermediate-containing serial positions 4 to 11 ; and recency - containing serial position 12. Choice of these grouping boundaries was based on visual inspection of serial position curves. We constructed two orthogonal dummy variables to evaluate differences between the three parts. The first variable, primacy, contrasted the primacy part with the mean of intermediate and recency parts; the second variable, recency, contrasted the intermediate with the recency part. Magnitude of these two contrasts was determined directly by the beta weights obtained from the model fits. Significance was evaluated with $t$ values. In large samples, $t$ distributions are approximately Gaussian, such that absolute $t$ values $>2.4$ indicate a Type I error probability of $p<$ .01 , which we took as the alpha level for significance in our post hoc tests and single predictor evaluations. The alpha level for model comparisons was set to $p<.05$. Effect sizes, $d$, of practicerelated improvements were calculated within each age group separately using the mean post-pretest differences normalized with the standard deviation at pretest. $^{2}$

Analysis of individual differences within and across measurement occasions. Individual differences in, and associations between, temporal and spatial accuracy measures at baseline and in practice-related change were analyzed using latent difference score models (see Figure 2; McArdle \& Nesselroade, 1994), a variant of structural equation modeling (Kline, 1998). In these models, latent pretest, change, and posttest factors were constructed on the basis of observed indicators, which, in our case, were obtained from odd-even splits of the trials at pretest and posttest. To better interpret differences at the latent level, metric invariance (constant factor loadings and intercepts) over time and groups is desirable (Meredith, 1964). In the context of measurement of change, this implies that the latent factors must not change with respect to their relation to the indicators. If metric invariance can be shown, latent variables can be interpreted as an expression of the true value of the indicators, free of measurement error (Meredith, 1964; see also McArdle \& Prindle, 2008). This feature is particularly helpful in the present study, as we are interested in the correlation between the gain scores for the temporal and spatial dimension. We tested for strong metric invariance on the spatial and temporal dimension separately, contrasting nested models with increasing constraints. The resulting metrically invariant models (with constant intercepts and factor loadings over time and groups as well as constant residual variances over time) showed reasonable model fit on the spatial, $\chi^{2}(12)=13$, RMSEA $=.03,90 \%$ CI $[.00, .11], C F I=$ 1.00 , and the temporal, $\chi^{2}(12)=20, R M S E A=.08,90 \%$ CI $[.00$, $.09], C F I=0.99$, dimension. Having this measurement model established, we estimated a bivariate latent difference score model, including covariances between the two dimensions (crossed), within each dimension over time (lagged), and between the two dimensions over time (cross-lagged). We analyzed group differences on each of these parameters by contrasting group homogenous against group heterogeneous models using likelihood ratio tests. Note that we tested homogeneity of correlations rather than covariances. Standardized parameter estimates are given with their corresponding $90 \%$ confidence intervals.

\section{Results}

\section{Group Differences and Changes}

For overall accuracy (see Table 2 for descriptive measures; Figure 3), we detected significant main effects of age, $\chi^{2}(1)=$ 107.47, $p<.05$, and session, $\chi^{2}(1)=93.35, p<.05$. Younger participants, $d=0.80$, gained more in accuracy over time than older, $d=0.59$, participants, $\chi^{2}(1)=20.26, p<.05$. The increase in performance was significant for both groups, $t(100)=8.05, p<$ .05 , for younger, and $t(102)=5.44, p<.05$, for older adults.

Next, we looked at the effects of spatial and temporal context in a linear model contrasting age, context dimension, and measurement time point. This model revealed a domain-general disadvantage in the old, $\chi^{2}(1)=110.00, p<.05$, but no reliable difference between temporal and spatial accuracy (see Table 2 ), $\chi^{2}(1)<1$. A strong interaction between age group and context, $\chi^{2}(1)=95.25$, $p<.05$, however, asked for more detailed follow-up analyses. First, we split the data set with respect to the context dimension, and analyzed the effect of age on temporal and spatial accuracy separately. Age differences were reliable on both dimensions (temporal: $\chi^{2}[1]=95.25, p<.05$; spatial: $\left.\chi^{2}[1]=25.58, p<.05\right)$. Second, we split the data set with respect to age group and analyzed the effects of context dimension on younger and older performance separately. We found reliable context effects in both age groups (younger: $\chi^{2}[1]=34.55, p<.05$; old: $\chi^{2}[1]=49.51$, $p<.05)$. Importantly, however, differences between spatial and temporal context pointed in opposite directions in the two age groups (see Figure 3), such that younger adults performed better on the temporal than on the spatial dimension, whereas older participants performed better on the spatial than on the temporal dimension. Taken together, this pattern of results implies that age-related differences were larger on the temporal than on the spatial dimension.

At a general level, training had a beneficial impact on accuracy, $\chi^{2}(1)=73.86, p<.05$. The magnitude of training gains varied, however, with age group, $\chi^{2}(1)=4.67, p<.05$, context dimension, $\chi^{2}(1)=9.70, p<.05$, and the interaction of the two, $\chi^{2}(1)=$ 5.96, $p<.05$. We looked again at the two age groups separately to locate the sources of these interactions. First, younger participants, $\chi^{2}(1)=21.08, p<.05$, and older participants, $\chi^{2}(1)=$

\footnotetext{
${ }^{2}$ Note that effect sizes for overall accuracy reported here differ from the effect sizes reported in Schmiedek, Bauer, et al. (2010). Differences are due to different procedures in data aggregation. Schmiedek et al. integrated mean spatial accuracy and a measure of temporal accuracy (Kendall's $\tau$ ) at the level of trials but here we determined overall accuracy at the level of single items (see Methods). Otherwise, calculation of effect sizes was identical with the procedures described by Schmiedek et al.
} 


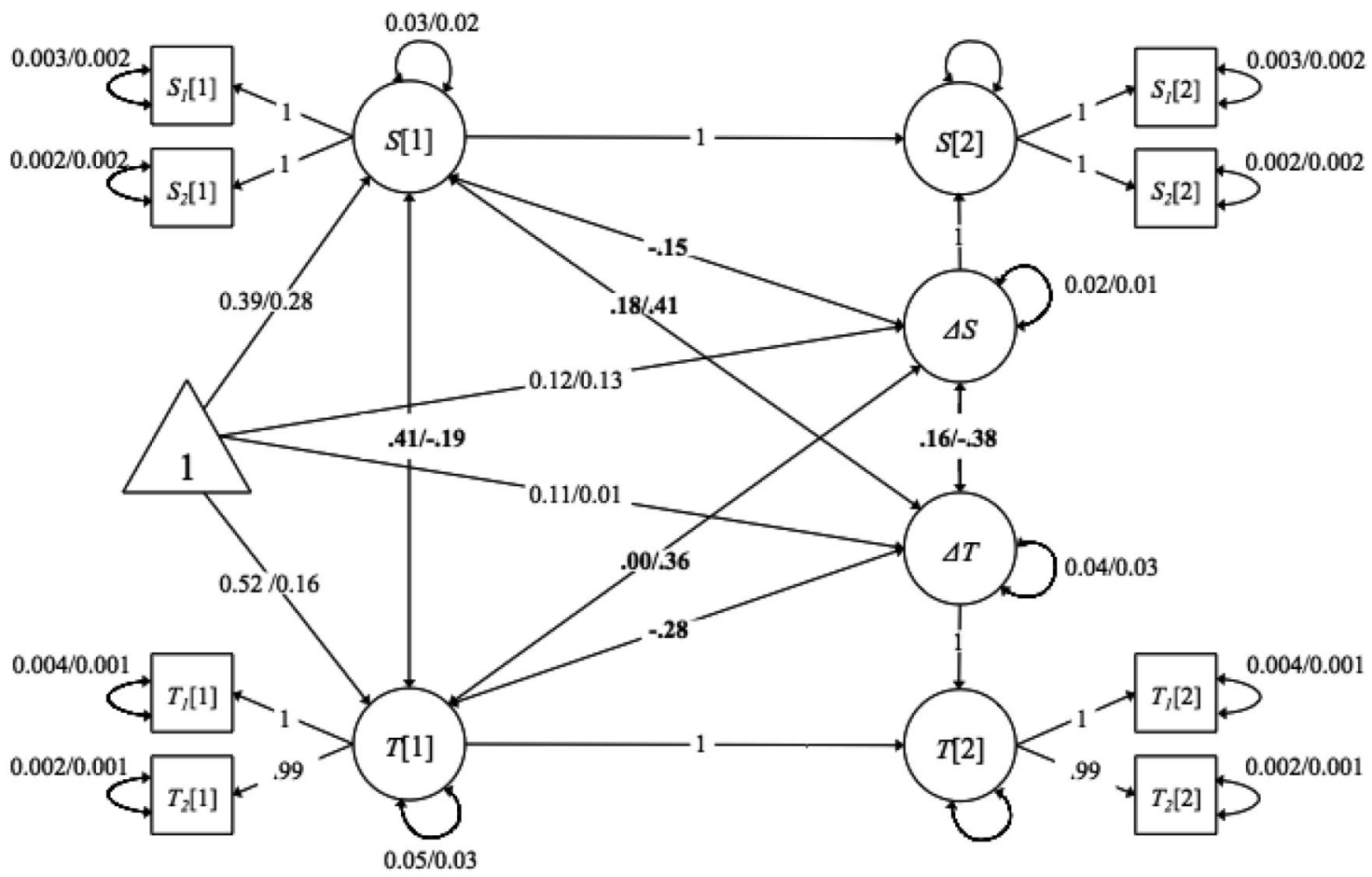

Figure 2. Bivariate latent change score model with common factors for spatial and temporal accuracy. The model is shown for one group only. Differences between the two groups are reflected in the path coefficients with coefficients of the younger group first and coefficients for the older group second. Only one path coefficient is shown on paths that did not differ between age groups. Note that standardized path coefficients are given for crossed, lagged, and cross-lagged correlations (bold face) and unstandardized path-coefficients are given for all other paths.

15.88, $p<.05$, improved on a domain-general level. However, although this improvement was true on each context dimension in the younger, $\left(\chi^{2}[1]<1\right.$; spatial: $d=0.72, t[100]=-7.80, p<$ .05 ; temporal: $d=0.46, t[100]=-5.18, p<.05)$, training gains differed between context dimensions in the old, $\chi^{2}(1)=13.83$, $p<.05$. Follow-up comparisons revealed a reliable improvement on the spatial dimension, $d=0.98, t(102)=-10.80, p<.05$, but no improvement on the temporal dimension, $d=0.09$; $|t(102)|<$ 1. In sum, older participants gained less than younger participants, both groups gained less on the temporal than on the spatial dimension, and, finally, younger participants gained on both dimensions, whereas improvement was restricted to the spatial dimension in the old.

To analyze how well accuracy measures in both context dimensions overlapped to form overall accuracy, we estimated an Age Group $\times$ Session model on the proportion of overall accurate trials within the set of trials where at least one dimension was remembered correctly. Age groups differed on this measure, $\chi^{2}(1)=$ 96.85, $p<.05$, performance increased with training, $\chi^{2}(1)=$ $36.82, p<.05$, and performance increased more for the younger than for the old, $\chi^{2}(1)=11.99 ; p<.05$. That is, in addition to lower memory on each separate dimension of the task, older

Table 2

Mean Accuracies (SDs)

\begin{tabular}{|c|c|c|c|c|c|c|c|}
\hline \multirow[b]{2}{*}{ Time } & \multirow[b]{2}{*}{ Serial position } & \multicolumn{3}{|c|}{ Younger adults } & \multicolumn{3}{|c|}{ Older adults } \\
\hline & & Overall & Spatial & Temporal & Overall & Spatial & Temporal \\
\hline \multirow[t]{4}{*}{ Pretest } & Early & $0.52(0.21)$ & $0.53(0.19)$ & $0.77(0.20)$ & $0.33(0.20)$ & $0.41(0.16)$ & $0.58(0.22)$ \\
\hline & Intermediate & $0.24(0.17)$ & $0.35(0.17)$ & $0.43(0.26)$ & $0.06(0.06)$ & $0.24(0.14)$ & $0.11(0.29)$ \\
\hline & Late & $0.24(0.19)$ & $0.35(0.20)$ & $0.47(0.29)$ & $0.09(0.13)$ & $0.31(0.22)$ & $0.19(0.29)$ \\
\hline & Total & $0.31(0.17)$ & $0.39(0.17)$ & $0.51(0.23)$ & $0.13(0.08)$ & $0.29(0.13)$ & $0.23(0.17)$ \\
\hline \multirow[t]{4}{*}{ Posttest } & Early & $0.58(0.25)$ & $0.59(0.22)$ & $0.79(0.33)$ & $0.41(0.24)$ & $0.53(0.16)$ & $0.55(0.34)$ \\
\hline & Intermediate & $0.41(0.26)$ & $0.49(0.23)$ & $0.57(0.14)$ & $0.10(0.11)$ & $0.37(0.16)$ & $0.14(0.19)$ \\
\hline & Late & $0.39(0.28)$ & $0.50(0.25)$ & $0.58(0.26)$ & $0.11(0.17)$ & $0.47(0.21)$ & $0.18(0.27)$ \\
\hline & Total & $0.45(0.25)$ & $0.52(0.22)$ & $0.62(0.26)$ & $0.18(0.13)$ & $0.41(0.15)$ & $0.24(0.21)$ \\
\hline
\end{tabular}


A

overall

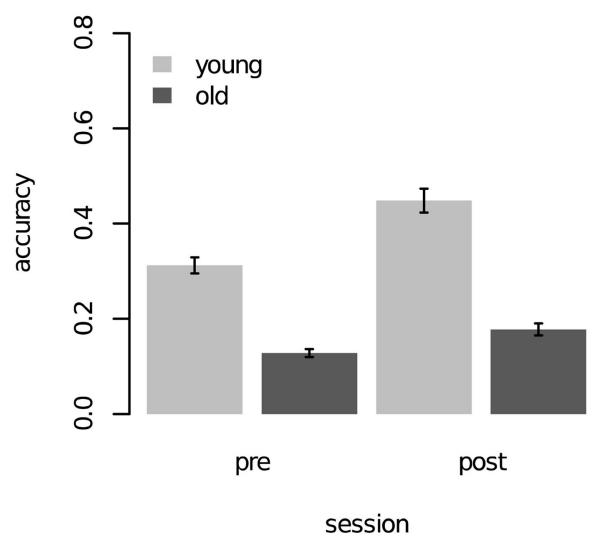

C

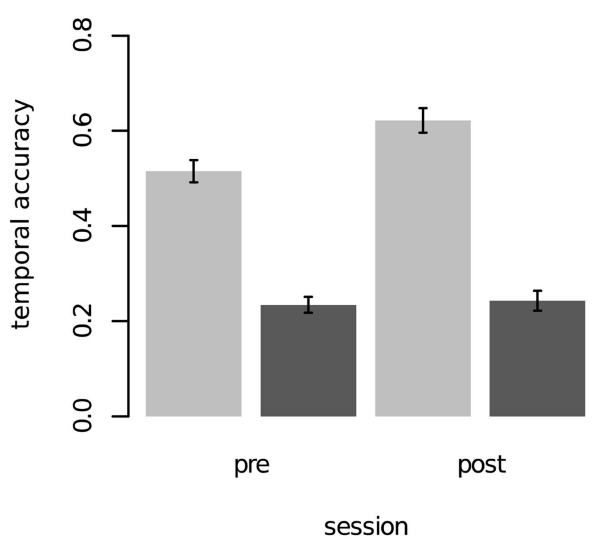

B

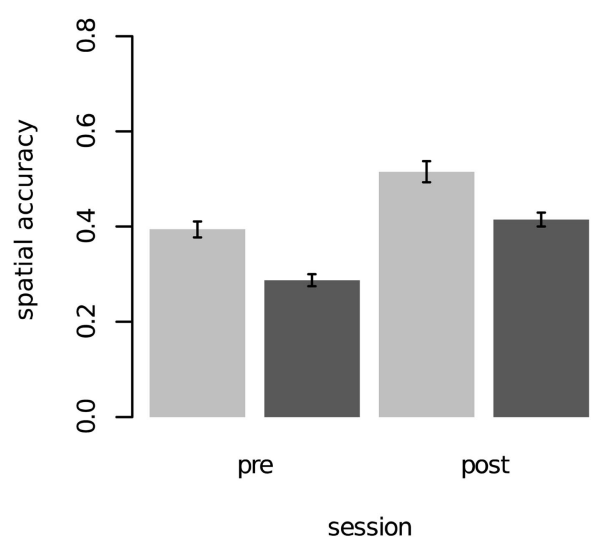

D

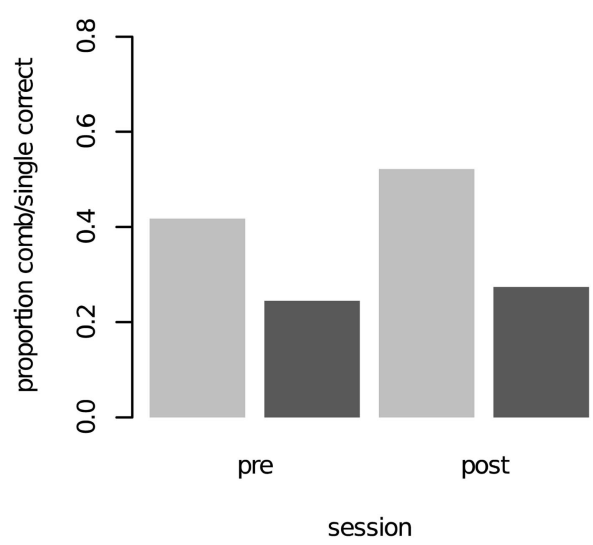

Figure 3. Mean group differences for (A) overall accuracy, i.e., correct object selected and placed at the correct spatial location; (B) spatial accuracy, i.e., object placed at original position; (C) temporal accuracy, i.e., object select at the correct serial position; (D) binding, i.e., proportion of number of trials with correct combination of serial position, object, and spatial location and number of trials of any correct response. Error bars represent \pm 1 standard error of the mean.

individuals showed less overlap in performance between the two dimensions and gained less from training in the overlap than younger adults.

\section{Individual Differences Within and Across Measurement Occasions}

The estimated bivariate latent change score factor model is depicted in Figure 2. We started with an invariant measurement model (see Methods for details) and an age-heterogeneous structure model, where lagged, crossed, and cross-lagged covariances were all free to vary across groups. This model fitted the data reasonably, $\chi^{2}(48)=91, R M S E A=.09,90 \%$ CI $[.06, .12], C F I=$ .98. To test for age differences in the relation between temporal and spatial dimension at pretest and in change, we constrained the correlations between the latent pretest scores and the latent change scores to be equal over age groups. Both constraints led to considerable decrements in model fit (pretest: $\mathrm{d} \chi^{2}[1]=19$; change: $\left.\mathrm{d} \chi^{2}[1]=17\right)$, indicating that correlations between latent pretest scores and correlations between latent change scores differed across age groups (see Figure 2). Notably, these correlations did not just differ in magnitude but differed in sign. That is, whereas younger participants showed a positive relationship between temporal and spatial accuracy at pretest, $r=.41,90 \%$ CI $[.27, .55]$, older people showed a low but reliably negative relationship, $r=$ $-.19,90 \%$ CI $[-.34,-.03]$. The pattern was similar at the level of latent difference scores: Older participants showed a negative relationship between the temporal and the spatial dimension, $r=$ $-.38,90 \% \mathrm{CI}=[-.53,-.24]$, whereas younger participants showed a trend in the positive direction, $r=.16,90 \% \mathrm{CI}=[.01$, .33].

To see how the change scores related to the initial levels, we also looked at the lagged (i.e., between pretest and change within one dimension) and cross-lagged (i.e., between pretest and change across dimensions) correlations. Again, we tested for age differences forcing the correlations to be equal across groups. Although lagged correlations did not differ between age groups, $\mathrm{d} \chi^{2}(2)=3$, cross-lagged correlations were significantly stronger in the old than in the young, $\mathrm{d} \chi^{2}(2)=8$. The resulting model with age 
homogeneous lagged and age heterogeneous crossed and crosslagged correlations still showed acceptable fit, $\chi^{2}(50)=94$, RMSEA $=.09,90 \% \mathrm{CI}=[.06, .12], C F I=.98$. Interestingly, parameter estimates revealed that change was negatively related to initial levels of the same dimension but mainly positively related to the initial levels of the other dimension (see Figure 2).

To graphically display this multivariate pattern of associations, we plotted pretest and posttest performance on the two dimensions as a two-dimensional vector field in Panel A of Figure 4. In this figure, points on the diagonal line denote individuals with equal performance on the spatial and temporal dimensions, points above the diagonal represent individuals with superior performance on the spatial dimension, and points below the diagonal represent individuals with superior performance on the temporal dimension. Points of pretest and posttest performance are connected by arrowheaded lines (vectors of change), which are characterized by amplitude (length of arrow) and direction (direction of arrow). The interplay between the two dimensions is best understood by focusing on the directions of change.

The change vectors of many younger participants point to the upper right corner of the plane. This suggests an effect of training on both the temporal and spatial dimension of the task. However, for the participants that are performing highly on either of the dimensions at pretest, improvements are restricted to the other dimension. This pattern is likely to explain the relatively low correlation between the difference scores, the positive correlation between pretest performance in one dimension and change in the other dimension, and the negative correlation between pretest performance and change on the temporal dimension.

In the older participants, however, Figure 4 reveals a more diverse pattern of change. In a kernel-density plot, which summarizes the directional frequency distribution of the change vectors, we found wider distributions for the old relative to the young (see Panel B in Figure 4). Whereas the distribution of younger participants is primarily located in the upper right quadrant, indicating increases on both dimensions, older people show an additional mode in the upper left quadrant. These directions indicate dimension-specific improvements or even trade-off with loss on the temporal and gain on the spatial dimension. This pattern is
A

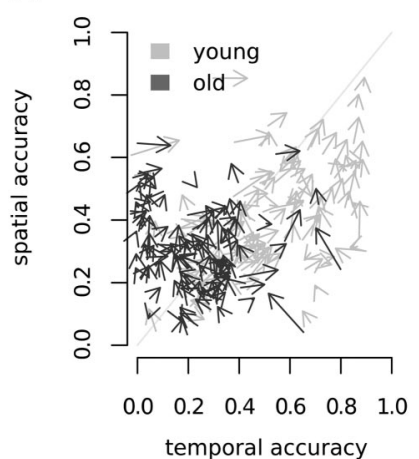

\section{B}

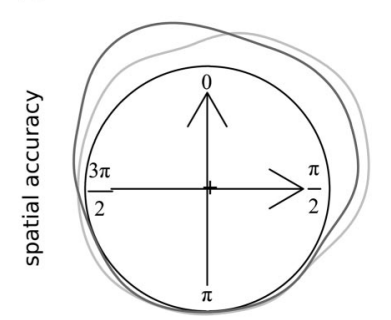

temporal accuracy
Figure 4. Change of temporal and spatial accuracy. (A) Vector field representing change on temporal and spatial dimension. Diagonal represents equal performance levels on both dimensions. (B) Kernel density $(\mathrm{bw}=20)$ plot of directions of change. consistent with the negative cross-lagged changes reported previously. Given the negative correlation between the dimensions at pretest, this shift toward spatial accuracy among the older adults also accounts for the negative correlations between pretest performance and change within one dimension and the positive correlations between pretest performance and change across dimensions. At the same time, the overlap in the directional density distributions of younger and older participants indicates that a dichotomous distinction between younger and older participants does not adequately describe the present results.

\section{Effects of Serial Position}

The analysis of serial position effects (see Figure 5) was based on the assumptions that (a) the ability to retain temporal order information should be reflected particularly in the curvature of the serial position curve for temporal accuracy, and (b) older participants, who have deficits representing item contiguity, should show a stronger curvature on the temporal relative to the spatial dimension. We tested these predictions in a model including the factors of age, context dimension, and serial position, with accuracy as the dependent measure. First, we obtained a strong main effect of serial position, $\chi^{2}(2)=858.62, p<.05$, which was further modulated by context dimension, $\chi^{2}(2)=317.65, p<.05$. In agreement with our first assumption, the primacy effect, that is, the difference between early ( 1 to 3 ) and the mean of intermediate ( 4 to 11) and late (12) list positions, was stronger on the temporal than on the spatial dimension, $\beta_{\text {primacy }} \times$ dimension $=0.035 ; t=$ 17.82. Recency effects, that is, the difference between the intermediate and the latest list position, did not differ between context dimensions, $|t|<1$. Separate analyses for spatial and temporal dimension revealed that serial position had a reliable impact on temporal accuracy, $\chi^{2}(2)=44.81, p<.05$, and on spatial accuracy, $\chi^{2}(2)=372.27, p<.05$, suggesting that representations of both spatial and temporal information varied by serial position. In agreement with our second assumption, we found a reliable interaction of age and serial position, $\chi^{2}(2)=56.57, p<.05$, which was further modulated by context dimension, $\chi^{2}(2)=49.21, p<$ .05 , such that older participants showed a particularly large primacy effect on the temporal dimension, $\beta_{\text {primacy }} \times$ dimension $\times$ age $=$ $0.012, t=6.67$. The triple interaction of age, context dimension, and recency effect was not reliable, $\beta_{\text {recency }} \times$ dimension $\times$ age $=$ $0.006, t=2.00$. Generally, then, our results confirmed our hypotheses, showing that serial position curves are particularly sensitive to the representation of temporal order, and suggesting that the ability to retain temporal order (i.e., contiguity) was particularly reduced in older participants. Note, however, that older participants showed lower accuracy on both, spatial, $t(202)=$ $3.85, p<.05$, and temporal, $t(202)=6.52, p<.05$, dimensions, also at early list positions. The increased primacy effect was therefore due to disproportionately low performance at intermediate and late list positions, rather than to disproportionately high primacy performance.

To see how training influenced this age-related difference, we looked at the effect of training on temporal and spatial accuracy at the three serial positions in younger and older adults. In line with the analysis of mean differences, we observed a reliable interaction between age, time, and context dimension, $\chi^{2}(1)=8.77, p<.05$, reflecting the small size of the increase in temporal accuracy 
A

pretest

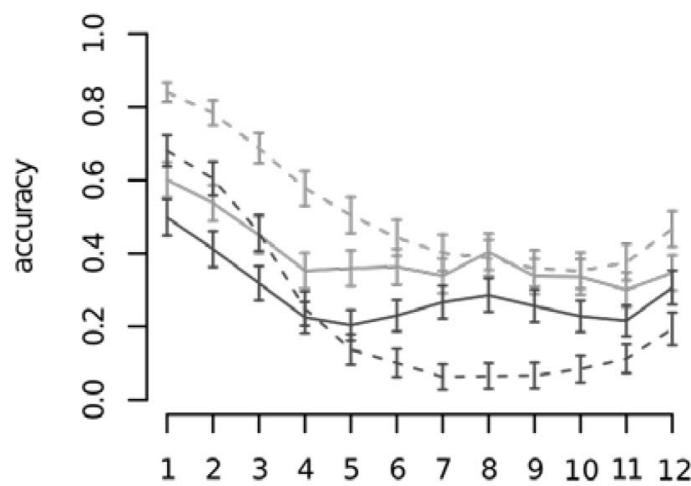

serial position

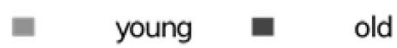

C

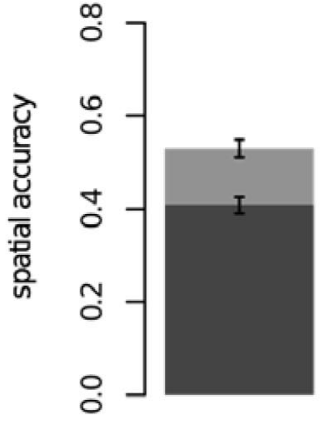

early

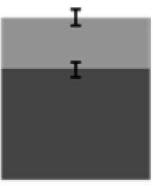

inter

serial position

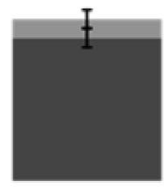

final

E

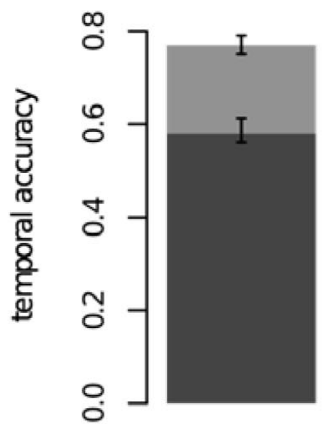

early

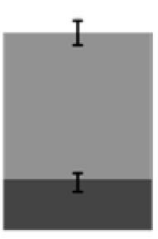

inter

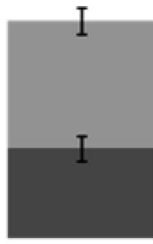

final
B

posttest

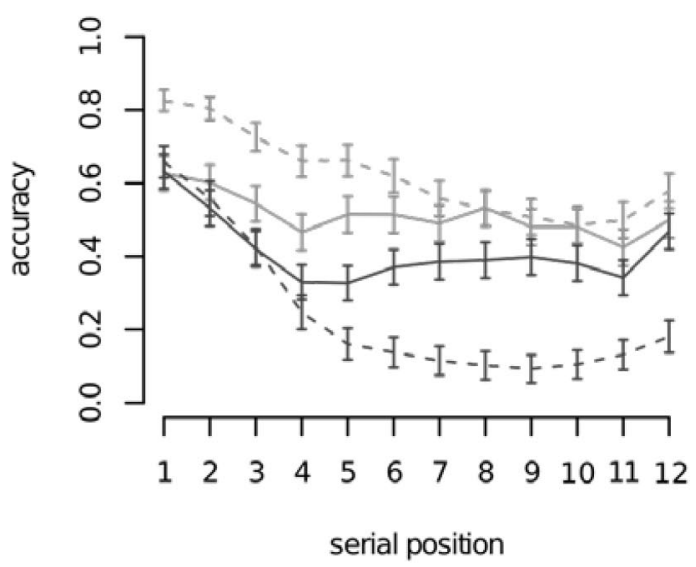

- spatial - - temporal

D

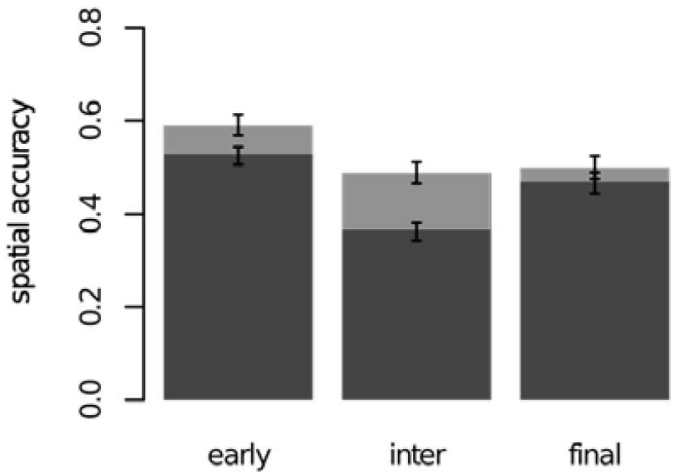

serial position

$\mathbf{F}$

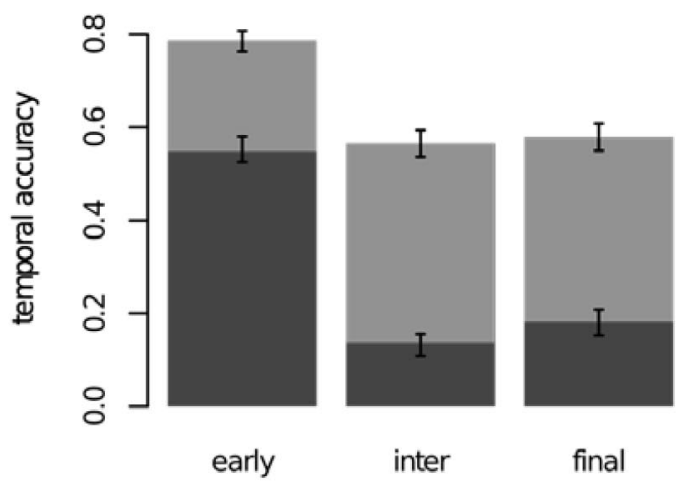

serial position

serial position

Figure 5. Serial position curves of spatial and temporal accuracy of younger and older adults are shown for (A) pretest and (B) posttest. Performance at three serial positions is shown for younger and older participants at pretest $(\mathrm{C}, \mathrm{E})$ and posttest $(\mathrm{D}, \mathrm{F})$ for spatial $(\mathrm{C}, \mathrm{D})$ and temporal $(\mathrm{E}, \mathrm{F})$ context dimension. Performance of younger participants is depicted in gray and performance of older participants is depicted in black, such that the difference between the two age groups can be taken from the gray area on top of each bar. Error bars denote \pm 1 standard error of the mean. 
among the older participants. This interaction, however, was not further modulated by serial position, $\chi^{2}(2)<1$. Instead, we observed a reliable three way interaction of age, serial position, and time, $\chi^{2}(2)=6.793, p<.05$. That is, irrespective of the context dimension, we found a marginally stronger reduction in the primacy effect in younger than in older adults, $\beta_{\text {primacy }} \times$ Time $\times$ age $=$ $-0.005, t=2.37$. Because accuracy at the early list positions increased or remained constant in the young (see Figure $5 \mathrm{c}$ through f), this effect can be attributed to greater increases in accuracy for the intermediate and the last serial positions. The recency effect did not vary by age and time, $|t|<1$. Taken together, these results extend the finding of age-related differences in improvements on the temporal context dimension, showing that these improvements originated particularly from intermediate serial positions.

\section{Metric Errors}

Figure 6 shows that the $95 \%$ confidence intervals of the mean spatial and temporal distance did not overlap with the estimates based on simulated data generated under the assumption of random error responses or strategic random responses on both context dimensions. Thus, younger and older participants committed errors that were closer in time and space to correct responses than expected by chance.

Looking at the difference between simulated and observed distances on the spatial dimension (Figure 6a), we found a significant age effect, $\chi^{2}(1)=37.67, p<.05$, indicating that older participants' error responses deviated less from random response levels than younger participants' responses. No further reliable effects were observed, $\chi^{2}(1)<4.5, p>.05$.

In the temporal dimension, we again observed age-related differences in distance to correct responses (Figure 6b). These differences were paralleled by a strong age-related difference in expected random displacement, which may reflect that younger participants recalled the first and last items better than older participants. Taking this difference into account, by using the difference between the expected random and the observed temporal displacement as a dependent measure, we did not find any reliable effects of age group or intervention, $\chi^{2} \mathrm{~s}(1)<3.12, p \mathrm{~s}>$ 0.05 . In summary, these findings suggest that erroneous responding on both context dimensions carries information about the original temporal or spatial coordinates of an item. Although age groups differ in this respect on the spatial dimension, no agerelated differences were detected on the temporal dimension.

\section{Discussion}

The present results replicate and extend findings of age-related decrements in temporal (Golomb et al., 2008; Kessels et al., 2007; Li et al., 2010; Maylor et al., 1999) and spatial (Chalfonte \& Johnson, 1996; Cherry \& Park, 1993; Kessels et al., 2007; Mitchell et al., 2000; Naveh-Benjamin, 1987; Puglisi et al., 1985) context memory. In contrast to previous direct comparisons of age-related differences in these types of context memory (Kessels et al., 2007), we observed larger age-related differences in performance for temporal context than for spatial context. Extensive general cognitive training and practice on the memory task improved overall performance. Younger adults gained more than older adults (see also Baltes \& Kliegl, 1992; Brehmer et al., 2007; Kliegl et al., 1989; Li et al., 2008; Schmiedek, Lövdén, et al., 2010; Shing et al., 2008; for reviews, see Lövdén, Bäckman, et al., 2010; Noack et al., 2009). The smaller improvement in overall accuracy for the older adults was mainly due to the absence of improvements on the temporal dimension, whereas younger and older participants improved similarly on the spatial dimension of the task.

The pattern of age differences in improvement from practice strongly suggests that the older adults were less able to simultaneously process both the temporal and spatial information of the items. The analyses of the associations between the two dimensions within and across age groups over practice lend further

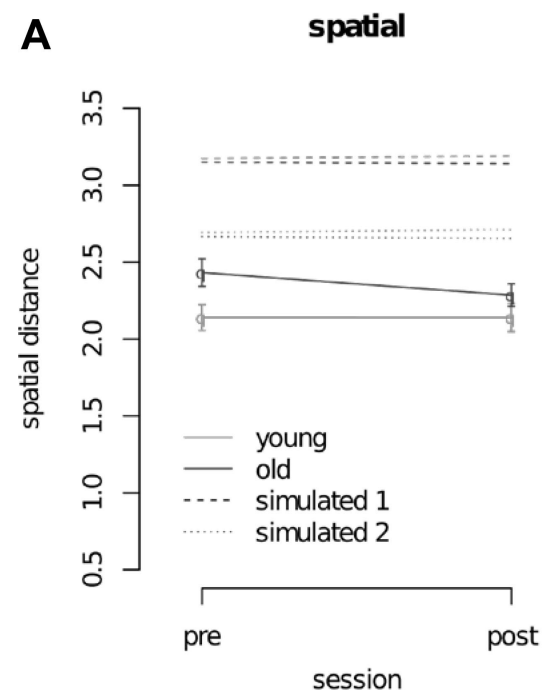

B

temporal

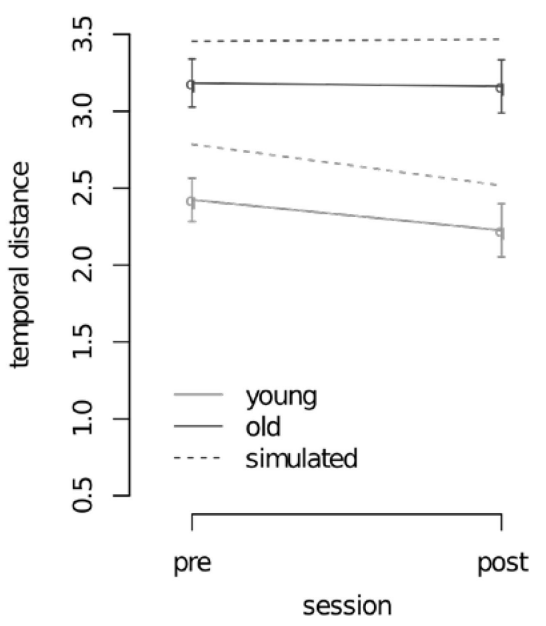

Figure 6. Spatial and temporal errors. (A) Observed and expected (simulated) mean Euclidean distances to original position. Simulated 1 represent mean expectations based on a completely random guessing and Simulated 2 represent mean expectations based on strategic guessing. (B) Absolute temporal position distance and random expected temporal distance (simulated). Error bars denote $\pm 1.96^{*}$ standard error of the mean. 
support to this interpretation. Younger participants showed a positive correlation between the two dimensions, indicating that baseline performance on the two dimensions was positively linked, and that performance improvements on the two dimensions moved in concert. In stark contrast, the negative correlations between the two dimensions in the group of older adults suggest that older participants tended to focus on one dimension while ignoring the other. These findings indicate that older participants, more than younger participants, had problems encoding both context dimensions at the same time, retrieving them at the same time, or both. This interpretation is in line with results obtained by Mitchell and colleagues (2000), who found that memory was disproportionately impaired in older adults relative to younger adults when participants were asked to recognize two isolated features (object + location) instead of one single feature.

The smaller practice-related gains for older adults in the temporal domain might be due to a preference of older adults to focus on the spatial dimension of the task. Such a differential focus may be driven by perceived differences in difficulty between the two dimensions. Though previous findings do not support the notion that temporal and spatial memory per se are differentially sensitive to aging (Parkin et al., 1995; Vakil \& Tweedy, 1994), differences in processing demands posed by the two context domains may make tasks or task dimensions that demand memory for temporal context more difficult and more age-sensitive than tasks or task dimensions that demand memory for spatial context. One potential source for differences in age sensitivity between the two dimensions may relate to the kind of associations required. For the temporal dimension, associations must be formed between the items in the sequence. On the spatial dimension, context associations can be formed for each item independently. This difference may have an effect particularly at retrieval, as the accuracy of temporal-order decisions depends on prior and subsequent decisions (Polyn \& Kahana, 2008; Ranganath, 2010), whereas the accuracy of spatial decisions is less dependent on prior and subsequent decisions. It has been shown that older people have particular problems using temporal contiguity to aid retrieval in free recall (Golomb et al., 2008; Kahana et al., 2002) and to reconstruct series of objects in serial recall (Golomb et al., 2008). Probably for this reason, age-related differences are particularly pronounced for intermediate and recency portions of the serial position curve ( $\mathrm{Li}$ et al., 2010). This pattern was also observed in the present data.

The present results also appear to be consistent with the general notion of an associative binding deficit in old age (Naveh-Benjamin, 2000; Old \& Naveh-Benjamin, 2008), as the proportion of entirely correct trials to the number of trials, where one of the two dimensions correctly recalled, was higher in younger adults than in older adults. However, this evidence should be interpreted with caution. In freerecall paradigms, estimates of binding are dependent on performance levels on the underlying context dimensions. Under the assumption of independent representations of temporal (T) and spatial (S) context, we would expect to observe a correct combination of the two with a probability of $p(\mathrm{~T} \wedge \mathrm{S})=p(\mathrm{~T}){ }^{*} p(\mathrm{~S})$. Similarly, for changes on only one dimension (e.g., $\mathrm{S})$, we find $\Delta p(\mathrm{~T} \wedge \mathrm{S})=p(\mathrm{~T}) *\left(p_{2}[\mathrm{~S}]-p_{1}[\mathrm{~S}]\right)$. That is, even if representations were independent, we would expect to observe correct bindings. Likewise, in the context of age-related differences in feature memory, we would expect age-related differences in correct feature combinations under the assumption of independent representations. Furthermore, it may not be sufficient to demonstrate equal performance levels for single-feature memory to overcome this problem because simultaneous retrieval of multiple features may induce additional age-related differences (Mitchell et al., 2000). For these reasons, the estimates of binding performance in the present study are open to alternative interpretations.

Temporally ordered decisions demand that the current state of action must be continually updated as one moves through the series. Neural correlates of memory updating and task management have been identified in the prefrontal cortex (PFC; Miller \& Cohen, 2001; Smith \& Jonides, 1999), suggesting that temporal context memory might be particularly dependent on the PFC. In agreement with this assertion, recent neuroimaging studies have revealed that the processing of interitem relations, such as on the temporal dimension, is modulated by dorsolateral prefrontal cortex (dIPFC) activity, whereas processing of item-specific context, such as on the present spatial dimension, is not (Blumenfeld, Parks, Yonelinas, \& Ranganath, 2011; Hsieh et al., 2011; Ranganath, 2010). Specifically, several lesion studies found that the dorsolateral (Kopelman et al., 1997), ventromedial (Szatkowska, Szymanska, \& Grabowska, 2004), and orbitofrontal PFC (Duarte et al., 2010) were associated with deficits in temporal-order processing but not with location processing (see also Milner, Corsi, \& Leonard, 1991; Petrides \& Milner, 1982; Shimamura, Janowsky, \& Squire, 1990). Similarly, Ekstrom and colleagues (2011) reported a more domain-specific dissociation, with greater prefrontal activation in a temporal than in a spatial retrieval task and greater parahippocampal activation in the spatial than in the temporal retrieval task. Temporal-order memory may thus impose greater demands on frontal lobe processing than spatial location processing because temporal-order memory critically depends on the organization of interitem associations (Blumenfeld et al., 2011; Polyn \& Kahana, 2008; Ranganath, 2010). In line with this notion, Parkin and colleagues (1995) found that temporal-order memory performance, but not spatial location memory performance, correlated with behavioral measures of frontal lobe functioning in older adults (see also Fabiani \& Friedman, 1997). Older adults have been shown to have particular deficits in tasks supposedly tapping the frontal lobes (Braver et al., 2001; Craik et al., 2010; Lindenberger et al., in press; West, 1996). Hence, age-related impairments may have been more pronounced for the temporal-order than for the spatial-location dimension because the processing of temporal order depends to a greater extent on the frontal lobes. In the same vein, the present results also suggest that normal aging may affect plasticity for temporal context memory more than plasticity for spatial location memory, perhaps again due to compromised frontal lobe processing. Note, however, that plasticity of frontal-lobe functions and structures is not entirely lost in old age (Lövdén, Bodammer, et al., 2010; Schmiedek, Lövdén, et al., 2010).

Finally, we extended the notion of partial source information (Dodson et al., 1998; Johnson et al., 1993) to the analysis of representations within single dimensions, assuming that representations of, for example, spatial locations have the form of probability distributions (Thurstone, 1927), which have a modal value over the original value of an item, but also include neighboring sites to some extent (see also Sanborn et al., 2010). If this idea were valid, then we would expect to see a considerable proportion of erroneous responses that are quite close to the original value. Note, however, that this view does not preclude the possibility that items are not encoded or retrieved successfully, which would then lead to random responding. In agreement with this proposition, we 
found that participants' erroneous responses were reliably more accurate than what would have been expected based on a random response model. Thus, it seems that participants knew more about the location of objects than was suggested by strict accuracy measures. Importantly, there was an age-related difference in the level of randomness observed, with older participants being closer to the level of expected random displacements on spatial accuracy. The source of this difference is difficult to establish based on the present data, as the difference between observed displacements and expected random displacements may be determined by two aspects: the dispersion of the representation and the level of failure to encode or retrieve an item (see, e.g., Xu \& Chun, 2009; Zhang \& Luck, 2008, for a similar account on working memory performance). Earlier studies have shown that older participants might show deficits on both of these aspects (Noack et al., 2012), but the present data do not allow us to separate these aspects.

Before concluding, we would like to remark on some limitations of the present study. First, one limiting aspect of the present study derives from the broadness of the training protocol. Participants received training on a broad set of different tasks, and we cannot exclude the possibility that the observed pattern of training gains resulted from spillover effects from other trained tasks rather than from the training of the object-memory task itself. Second, we did not study age-related performance differences in spatial or temporal memory alone. It is therefore difficult to separate the effect of potential age-related deficits in spatial and temporal memory alone from the effect of age on parallel processing performance. Finally, we framed our study in the conceptual context of episodic memory (Tulving, 1983, 2002) because this concept emphasizes the associations between content, time, and space. In that sense, our paradigm represents an exemplar of an episodic memory task. We acknowledge, however, that the concept is going beyond the idea of a tripartite representation system, implying, for example, autonoetic consciousness. Given that autonoetic consciousness was not necessary to master our task successfully, generalizability of the present results to other episodic memory tasks may be limited.

In summary, we observed larger age-related differences in performance for temporal context than for spatial context on a serial-order object-location memory task. Younger adults gained more than older adults from extensive general cognitive training and practice on this task. This age-related difference was mainly due to the absence of improvements on the temporal dimension for older adults. Younger participants showed positive correlations between the two dimensions, indicating that baseline performance and improvements on performance on both dimensions were, to some degree, integrated. Importantly, the corresponding correlations among older adults were negative, indicating that older participants tended to focus on one dimension while ignoring the other. These results suggest that normal aging impairs memory for temporal order to a greater extent than memory for spatial location and compromises the ability to focus simultaneously on both dimensions.

\section{References}

Baltes, P. B., \& Kliegl, R. (1992). Further testing of limits of cognitive plasticity: Negative age-differences in a mnemonic skill are robust. Developmental Psychology, 28, 121-125. doi:10.1037/0012-1649.28.1 .121
Bates, D. M., \& Maechler, M. (2010). lme4: Linear mixed-effects models using S4 classes. Version R package version 0.999375-39. Retrieved from http://R-Forge.R-project.org/projects/lme4/

Bayen, U. J., Phelps, M. P., \& Spaniol, J. (2000). Age-related differences in the use of contextual information in recognition memory: A global matching approach. Journals of Gerontology: Series B: Psychological Sciences and Social Sciences, 55, P131-P141. doi:10.1093/geronb/55.3 .P131

Blumenfeld, R. S., Parks, C. M., Yonelinas, A. P., \& Ranganath, C. (2011). Putting the pieces together: The role of dorsolateral prefrontal cortex in relational memory encoding. Journal of Cognitive Neuroscience, 23, 257-265. doi:10.1162/jocn.2010.21459

Braver, T. S., Barch, D. M., Keys, B. A., Carter, C. S., Cohen, J. D., Kaye, J. A., . . . Reed, B. R. (2001). Context processing in older adults: Evidence for a theory relating cognitive control to neurobiology in healthy aging. Journal of Experimental Psychology: General, 130, 746763. doi:10.1037/0096-3445.130.4.746

Brehmer, Y., Li, S. C., Müller, V., von Oertzen, T., \& Lindenberger, U. (2007). Memory plasticity across the life span: Uncovering children's latent potential. Developmental Psychology, 43, 465-478. doi:10.1037/ 0012-1649.43.2.465

Brown, G. D. A., \& McCormack, T. (2006). The role of time in human memory and binding: A review of the evidence. In H. D. Zimmer, A. Mecklinger, \& U. Lindenberger (Eds.), Handbook of binding and memory: Perspectives from cognitive neuroscience (pp. 251-290). New York, NY: Oxford.

Cansino, S. (2009). Episodic memory decay along the adult lifespan: A review of behavioral and neurophysiological evidence. International Journal of Psychophysiology, 71, 64-69. doi:10.1016/j.ijpsycho.2008 .07 .005

Chalfonte, B. L., \& Johnson, M. K. (1996). Feature memory and binding in young and older adults. Memory \& Cognition, 24, 403-416. doi: 10.3758/BF03200930

Cherry, K. E., \& Park, D. C. (1993). Individual difference and contextual variables influence spatial memory in younger and older adults. Psychology and Aging, 8, 517-526. doi:10.1037/0882-7974.8.4.517

Craik, F. I., Luo, L., \& Sakuta, Y. (2010). Effects of aging and divided attention on memory for items and their contexts. Psychology and Aging, 25, 968-979. doi:10.1037/a0020276

de Frias, C. M., Lövdén, M., Lindenberger, U., \& Nilsson, L. G. (2007). Revisiting the dedifferentiation hypothesis with longitudinal multicohort data. Intelligence, 35, 381-392. doi:10.1016/j.intell.2006.07.011

Dodson, C. S., Holland, P. W., \& Shimamura, A. P. (1998). On the recollection of specific- and partial-source information. Journal of Experimental Psychology: Learning, Memory, and Cognition, 24, 11211136. doi:10.1037/0278-7393.24.5.1121

Duarte, A., Henson, R. N., Knight, R. T., Emery, T., \& Graham, K. S. (2010). Orbito-frontal cortex is necessary for temporal context memory. Journal of Cognitive Neuroscience, 22, 1819-1831. doi:10.1162/jocn .2009 .21316

Dutta, A., \& Nairne, J. S. (1993). The separability of space and time: Dimensional interaction in the memory trace. Memory \& Cognition, 21, 440-448. doi:10.3758/BF03197175

Ekstrom, A. D., Copara, M. S., Isham, E. A., Wang, W. C., \& Yonelinas, A. P. (2011). Dissociable networks involved in spatial and temporal order source retrieval. NeuroImage, 56, 1803-1813. doi:10.1016/j .neuroimage.2011.02.033

Fabiani, M., \& Friedman, D. (1997). Dissociations between memory for temporal order and recognition memory in aging. Neuropsychologia, 35, 129-141. doi:10.1016/S0028-3932(96)00073-5

Gallistel, C. R. (1990). The organization of learning. Cambridge, MA: MIT Press.

Golomb, J. D., Peelle, J. E., Addis, K. M., Kahana, M. J., \& Wingfield, A. (2008). Effects of adult aging on utilization of temporal and semantic 
associations during free and serial recall. Memory \& Cognition, 36, 947-956. doi:10.3758/MC.36.5.947

Hälbig, T. D., Mecklinger, A., Schriefers, H., \& Friederici, A. D. (1998). Double dissociation of processing temporal and spatial information in working memory. Neuropsychologia, 36, 305-311. doi:10.1016/S00283932(97)00127-9

Hasher, L., \& Zacks, R. T. (1979). Automatic and effortful processes in memory. Journal of Experimental Psychology: General, 108, 356-388. doi:10.1037/0096-3445.108.3.356

Hertzog, C., Kramer, A. F., Wilson, R. S., \& Lindenberger, U. (2009). Enrichment effects on adult cognitive development: Can the functional capacity of older adults be preserved and enhanced? Psychological Science in the Public Interest, 9, 1-65. doi:0.1111/j.1539-6053.2009 .01034.x

Howard, M. W., Kahana, M. J., \& Wingfield, A. (2006). Aging and contextual binding: Modeling recency and lag recency effects with the temporal context model. Psychonomic Bulletin \& Review, 13, 439-445. doi:10.3758/BF03193867

Hsieh, L.-T., Ekstrom, A. D., \& Ranganath, C. (2011). Neural oscillations associated with item and temporal order maintenance in working memory. The Journal of Neuroscience, 31, 10803-10810. doi:10.1523/ JNEUROSCI.0828-11.2011

Johnson, M. K., Hashtroudi, S., \& Lindsay, D. S. (1993). Source monitoring. Psychological Bulletin, 114, 3-28. doi:10.1037/0033-2909.114.1.3

Kahana, M. J., Howard, M. W., Zaromb, F., \& Wingfield, A. (2002). Age dissociates recency and lag recency effects in free recall. Journal of Experimental Psychology: Learning, Memory, and Cognition, 28, 530540. doi:10.1037/0278-7393.28.3.530

Kahneman, D., Treisman, A., \& Gibbs, B. J. (1992). The reviewing of object files: Object-specific integration and information. Cognitive Psychology, 24, 175-219. doi:10.1016/0010-0285(92)90007-O

Kessels, R. P., Hobbel, D., \& Postma, A. (2007). Aging, context memory and binding: A comparison of "what, where and when" in young and older adults. International Journal of Neuroscience, 117, 795-810. doi:10.1080/00207450600910218

Kliegl, R., Smith, J., \& Baltes, P. B. (1989). Testing-the-limits and the study of adult age-differences in cognitive plasticity of a mnemonic skill. Developmental Psychology, 25, 247-256. doi:10.1037/0012-1649.25.2 .247

Kline, R. B. (1998). Principles and practice of structural equation modeling. New York, NY: Guilford Press.

Kopelman, M. D., Stanhope, N., \& Kingsley, D. (1997). Temporal and spatial context memory in patients with focal frontal, temporal lobe, and diencephalic lesions. Neuropsychologia, 35, 1533-1545. doi:10.1016/ S0028-3932(97)00076-6

Lewandowsky, S., Brown, G. D. A., Wright, T., \& Nimmo, L. M. (2006). Timeless memory: Evidence against temporal distinctiveness models of short-term memory for serial order. Journal of Memory and Language, 54, 20-38. doi:10.1016/j.jml.2005.08.004

Lewandowsky, S., \& Farrell, S. (2008). Short-term memory: New data and a model. Journal of Learning and Motivation, 49, 1-49. doi:10.1016/ S0079-7421(08)00001-7

Li, S.-C., Chicherio, C., Nyberg, L., von Oertzen, T., Nagel, I. E., Papenberg, G., . . Backman, L. (2010). Ebbinghaus revisited: Influences of the BDNF Val66Met polymorphism on backward serial recall are modulated by human aging. Journal of Cognitive Neuroscience, 22, 21642173. doi:10.1162/jocn.2009.21374

Li, S.-C., Schmiedek, F., Huxhold, O., Röcke, C., Smith, J., \& Lindenberger, U. (2008). Working memory plasticity in old age: Practice gain, transfer, and maintenance. Psychology and Aging, 23, 731-742. doi: $10.1037 / \mathrm{a} 0014343$

Light, L. L. (1991). Memory and aging: 4 hypotheses in search of data. Annual Review of Psychology, 42, 333-376. doi:10.1146/annurev.ps.42 .020191 .002001
Lindenberger, U., Burzynska, A. Z, \& Nagel, I. E. (in press). Heterogeneity in frontal-lobe aging. In D. T. Stuss \& R. T. Knight (Eds.), Principles of frontal-lobe functions. New York: Oxford University Press.

Lövdén, M., Bäckman, L., Lindenberger, U., Schaefer, S., \& Schmiedek, F. (2010). A theoretical framework for the study of adult cognitive plasticity. Psychological Bulletin, 136, 659-676. doi:10.1037/a0020080

Lövdén, M., Bodammer, N. C., Kühn, S., Kaufmann, J., Schütze, H., Tempelmann, C., . . . Lindenberger, U. (2010). Experience-dependent plasticity of white-matter microstructure extends into old age. Neuropsychologia, 48, 3878-3883. doi:10.1016/j.neuropsychologia.2010.08 .026

Maylor, E. A., Vousden, J. I., \& Brown, G. D. (1999). Adult age differences in short-term memory for serial order: Data and a model. Psychology and Aging, 14, 572-594. doi:10.1037/0882-7974.14.4.572

McArdle, J. J, \& Nesselroade, J. R. (1994). Using multivariate data to structure developmental change. In S. H. Reese \& H. W. Cohen (Eds.), Life-span developmental psychology: Methodological contributions (pp. 223-264). Hillsdale, NJ: Erlbaum.

McArdle, J. J., \& Prindle, J. J. (2008). A latent change score analysis of a randomized clinical trial in reasoning training. Psychology and Aging, 23, 702-719. doi:10.1037/a0014349

Meredith, W. (1964). Notes on factorial invariance. Psychometrika, 29 , 177-185. doi:10.1007/BF02289699

Meulenbroek, O., Kessels, R. P., de Rover, M., Petersson, K. M., Rikkert, M. G., Rijpkema, M., \& Fernandez, G. (2010). Age-effects on associative object-location memory. Brain Research, 1315, 100-110. doi: 10.1016/j.brainres.2009.12.011

Miller, E. K., \& Cohen, J. D. (2001). An integrative theory of prefrontal cortex function. Annual Review of Neuroscience, 24, 167-202. doi: 10.1146/annurev.neuro.24.1.167

Milner, B., Corsi, P., \& Leonard, G. (1991). Frontal-lobe contribution to recency judgments. Neuropsychologia, 29, 601-618. doi:10.1016/00283932(91)90013-X

Mitchell, K. J., Johnson, M. K., Raye, C. L., Mather, M., \& D’Esposito, M. (2000). Aging and reflective processes of working memory: Binding and test load deficits. Psychology and Aging, 15, 527-541. doi:10.1037/ 0882-7974.15.3.527

Naveh-Benjamin, M. (1987). Coding of spatial location information: An automatic process? Journal of Experimental Psychology: Learning, Memory, and Cognition, 13, 595-605. doi:10.1037/0278-7393.13.4.595

Naveh-Benjamin, M. (1988). Recognition memory of spatial location information: Another failure to support automaticity. Memory \& Cognition, 16, 437-445. doi:10.3758/BF03214224

Naveh-Benjamin, M. (1990). Coding of temporal order information: An automatic process? Journal of Experimental Psychology: Learning, Memory, and Cognition, 16, 117-126. doi:10.1037/0278-7393.16.1.117

Naveh-Benjamin, M. (2000). Adult age differences in memory performance: Tests of an associative deficit hypothesis. Journal of Experimental Psychology: Learning Memory and Cognition, 26, 1170-1187. doi: 10.1037/0278-7393.26.5.1170

Naveh-Benjamin, M., Cowan, N., Kilb, A., \& Chen, Z. (2007). Age-related differences in immediate serial recall: Dissociating chunk formation and capacity. Memory \& Cognition, 35, 724-737. doi:10.3758/BF03193310

Naveh-Benjamin, M., Shing, Y. L., Kilb, A., Werkle-Bergner, M., Lindenberger, U., \& Li, S. C. (2009). Adult age differences in memory for name-face associations: The effects of intentional and incidental learning. Memory, 17, 220-232. doi:10.1080/09658210802222183

Nilsson, L. G., Bäckman, L., Erngrund, K., Nyberg, L., Adolfson, R., Bucht, G., ... Windblad, B. (1997). The betula prospective cohort study: Memory health, and aging. Aging, Neuropsychology, and Cognition, 4, 1-32. doi:10.1080/13825589708256633

Noack, H., Lövdén, M., \& Lindenberger, U. (2012). Normal aging increases discriminal dispersion in visuo-spatial short-term memory. Psychology and Aging, 27, 627-637. doi:10.1037/a0027251 
Noack, H., Lövdén, M., Schmiedek, F., \& Lindenberger, U. (2009). Cognitive plasticity in adulthood and old age: Gauging the generality of cognitive intervention effects. Restorative Neurology and Neuroscience, 27, 435-453. doi:10.3233/RNN-2009-0496

Oberauer, K., \& Lewandowsky, S. (2008). Forgetting in immediate serial recall: Decay, temporal distinctiveness, or interference? Psychological Review, 115, 544-576. doi:10.1037/0033-295X.115.3.544

Old, S. R., \& Naveh-Benjamin, M. (2008). Differential effects of age on item and associative measures of memory: A meta-analysis. Psychology and Aging, 23, 104-118. doi:10.1037/0882-7974.23.1.104

Olson, I. R., Zhang, J. X., Mitchell, K. J., Johnson, M. K., Bloise, S. M., \& Higgins, J. A. (2004). Preserved spatial memory over brief intervals in older adults. Psychology and Aging, 19, 310-317. doi:10.1037/08827974.19.2.310

Parkin, A. J., Walter, B. M., \& Hunkin, N. M. (1995). Relationships between normal aging, frontal lobe function, and memory for temporal and spatial information. Neuropsychology, 9, 304-312. doi:10.1037/ 0894-4105.9.3.304

Perlmutter, M., Metzger, R., Nezworski, T., \& Miller, K. (1981). Spatial and temporal memory in 20 to 60 year olds. Journal of Gerontology, 36, 59-65. doi:10.1093/geronj/36.1.59

Petrides, M., \& Milner, B. (1982). Deficits on subject-ordered tasks after frontal- and temporal-lobe lesions in man. Neuropsychologia, 20, 249262. doi:10.1016/0028-3932(82)90100-2

Polyn, S. M., \& Kahana, M. J. (2008). Memory search and the neural representation of context. Trends in Cognitive Sciences, 12, 24-30. doi:10.1016/j.tics.2007.10.010

Puglisi, J. T., Park, D. C., Smith, A. D., \& Hill, G. W. (1985). Memory for two types of spatial location: Effects of instructions, age, and format. American Journal of Psychology, 98, 101-118. doi:10.2307/1422770

Ranganath, C. (2010). Binding items and contexts: The cognitive neuroscience of episodic memory. Current Directions in Psychological Science, 19, 131-137. doi:10.1177/0963721410368805

R Development Core Team. (2010). R: A language and environment for statistical computing. Vienna, Austria: Foundation for Statistical Computing. Retrieved from http://R-project.org

Richardson, J. T. (2007). Measures of short-term memory: A historical review. Cortex: A Journal Devoted to the Study of the Nervous System and Behavior, 43, 635-650. doi:10.1016/S0010-9452(08)70493-3

Rönnlund, M., Nyberg, L., Bäckman, L., \& Nilsson, L. G. (2005). Stability, growth, and decline in adult life span development of declarative memory: Cross-sectional and longitudinal data from a population-based study. Psychology and Aging, 20, 3-18. doi:10.1037/0882-7974.20.1.3

Salthouse, T. A. (1998). Independence of age-related influences on cognitive abilities across the life span. Developmental Psychology, 34, 851-864. doi:10.1037/0012-1649.34.5.851

Sanborn, A. N., Griffiths, T. L., \& Shiffrin, R. M. (2010). Uncovering mental representations with Markov chain Monte Carlo. Cognitive Psychology, 60, 63-106. doi:10.1016/j.cogpsych.2009.07.001

Schmiedek, F., Bauer, C., Lövdén, M., Brose, A., \& Lindenberger, U. (2010). Cognitive enrichment in old age: Web-based training programs. GeroPsych: The Journal of Gerontopsychology and Geriatric Psychiatry, 23, 59-67. doi:10.1024/1662-9647/a000013

Schmiedek, F., Lövdén, M., \& Lindenberger, U. (2010). Hundred days of cognitive training enhance broad cognitive abilities in adulthood: Findings from the COGITO study. Frontiers in Aging Neuroscience, 2. doi:10.3389/fnagi.2010.00027

Shimamura, A. P., Janowsky, J. S., \& Squire, L. R. (1990). Memory for the temporal order of events in patients with frontal lobe lesions and amnesic patients. Neuropsychologia, 28, 803-813. doi:10.1016/00283932(90)90004-8
Shing, Y. L., Werkle-Bergner, M., Brehmer, Y., Mueller, V., Li, S.-C., \& Lindenberger, U. (2010). Episodic memory across the lifespan: The contributions of associative and strategic components. Neuroscience and Biobehavioral Reviews, 34, 1080-1091. doi:10.1016/j.neubiorev.2009 .11 .002

Shing, Y. L., Werkle-Bergner, M., Li, S. C., \& Lindenberger, U. (2008). Associative and strategic components of episodic memory: A life-span dissociation. Journal of Experimental Psychology: General, 137, 495513. doi:10.1037/0096-3445.137.3.495

Simons, J. S., Dodson, C. S., Bell, D., \& Schacter, D. L. (2004). Specificand partial-source memory: Effects of aging. Psychology and Aging, 19, 689-694. doi:10.1037/0882-7974.19.4.689

Slotnick, S. D., \& Dodson, C. S. (2005). Support for a continuous (singleprocess) model of recognition memory and source memory. Memory \& Cognition, 33, 151-170. doi:10.3758/BF03195305

Smith, E. E., \& Jonides, J. (1999). Neuroscience: Storage and executive processes in the frontal lobes. Science, 283, 1657-1661. doi:10.1126/ science.283.5408.1657

Spencer, W. D., \& Raz, N. (1994). Memory for facts, source, and context: Can frontal lobe dysfunction explain age-related differences? Psychology and Aging, 9, 149-159. doi:10.1037/0882-7974.9.1.149

Spencer, W. D., \& Raz, N. (1995). Differential effects of aging on memory for content and context: A meta-analysis. Psychology and Aging, 10, 527-539. doi:10.1037/0882-7974.10.4.527

Szatkowska, I., Szymanska, O., \& Grabowska, A. (2004). The role of the human ventromedial prefrontal cortex in memory for contextual information. Neuroscience Letters, 364, 71-75. doi:10.1016/j.neulet.2004.03 .084

Thurstone, L. L. (1927). Psychophysical analysis. The American Journal of Psychology, 38, 368-389. doi:10.2307/1415006

Tulving, E. (1983). Elements of episodic memory. Oxford, UK: Clarendon Press.

Tulving, E. (2002). Episodic memory: From mind to brain. Annual Reviews of Psychology, 53, 1-25. doi:10.1146/annurev.psych.53.100901.135114

Vakil, E., \& Tweedy, J. R. (1994). Memory for temporal order and spatial position information. Neuropsychiatry, Neuropsychology, \& Behavioral Neurology, 7, 281-288.

Vakil, E., Weise, M., \& Enbar, S. (1997). Direct and indirect memory measures of temporal order: Younger versus older adults. The International Journal of Aging \& Human Development, 45, 195-206. doi: 10.2190/N54R-9Q1M-27F3-GTRY

van Asselen, M., Van der Lubbe, R. H., \& Postma, A. (2006). Are space and time automatically integrated in episodic memory? Memory, 14, 232-240. doi:10.1080/09658210500172839

West, R. L. (1996). An application of prefrontal cortex function theory to cognitive aging. Psychological Bulletin, 120, 272-292. doi:10.1037/ 0033-2909.120.2.272

Wickens, T. D. (2002). Elementary signal detection theory. Oxford, UK Oxford University Press.

Xu, Y., \& Chun, M. M. (2009). Selecting and perceiving multiple visual objects. Trends in Cognitive Sciences, 13, 167-174. doi:10.1016/j.tics .2009.01.008

Zhang, W., \& Luck, S. J. (2008). Discrete fixed-resolution representations in visual working memory. Nature, 453, 233-235. doi:10.1038/ nature 06860

Received July 9, 2012

Revision received October 22, 2012 Accepted November 21, 2012 